\title{
Forecast Precision and Forecast Accuracy from Moving Average and Moving Median Methods on Skewed Lognormal Time Series
}

\author{
Louie Ren \\ University of Houston-Victoria \\ Peter Ren \\ University of Houston-Downtown
}

We examine the forecast precision and accuracy for forecasts from moving average and moving median methods on skewed i.i.d. time series following various lognormal probability distributions. Overall, we recommend the Moving Average method, MA, when forecasting time series that follow lognormal distributions.

\section{INTRODUCTION}

In forecasting, "precision" measures the un-biasness of a forecasting method and "accuracy" measures the dispersions of a forecasting method. In absolute terms, the sample mean for the error terms, $\bar{e}$, and the sample standard deviation for the error terms, $\mathrm{s}_{\mathrm{e}}$, are the most popular measures for forecast precision and accuracy, respectively. The inverse coefficient of variation, $\frac{\bar{e}}{s_{e}}$ (Hoover, 2006), and the coefficient of variation, $\frac{s_{e}}{\bar{e}}$ (Anderson, Sweeney, Williams, Camm, and Cochran 2016), are the two most popular scale-free measures for forecast precision and accuracy, respectively.

In this study, we introduce a new forecast measure from the linear combination of scale-free forecast measures:

$$
\mathrm{L}_{\omega}=\omega\left(\frac{\bar{e}}{s_{e}}\right)+(1-\omega)\left(\frac{s_{e}}{\bar{e}}\right), \text { where } 0 \leq \omega \leq 1
$$

The weight, $\omega$, serves to reflect the forecaster's emphasis on precision relative to accuracy. When $\omega=$ $0, \mathrm{~L}_{\omega=0}$ is the popular scale free forecast accuracy measure, the coefficient of variation, $\frac{s_{e}}{\bar{e}}$. When $\omega=1$, $\mathrm{L}_{\omega=1}$ is the popular scale free forecast accuracy measure, the inverse coefficient of variation, $\frac{\bar{e}}{s_{e}}$. For instance, when $\omega=0.2$, the forecaster is more concerned (80\%) about the forecast accuracy from $\frac{s_{e}}{\bar{e}}$ rather than the forecast precision from $\frac{\bar{e}}{s_{e}}$.

In this study, we compare forecast precisions and accuracies from the forecasting methods Moving Average (MA) and Moving Median (MMd) on various skewed i.i.d. time series following lognormal 
distributions. Forecast precisions and accuracies are measured by absolute measures, $\bar{e}$ and $\mathrm{s}_{\mathrm{e}}$, and relative measures $\mathrm{L}_{\omega}$ for $\omega=0,0.2,0.4,0.6,0.8$, and, 1.0 .

We find that out of skewed i.i.d. time series from lognormal distributions with parameters $(\mu=0, \sigma$ $=1),(\mu=0, \sigma=0.5),(\mu=0, \sigma=0.25),(\mu=0, \sigma=0.1)$ and skewness $\left(e^{\sigma^{2}}+2\right) \sqrt{e^{\sigma^{2}}-1}, 6.1849,1.9446$, $0.8340,0.3113$, respectively, the Moving Median method, MMd, only outperforms the Moving Average method, MA, in terms of forecasting accuracy for extremely skewed lognormal distribution with parameters $(\mu=0, \sigma=1)$, and skewness 6.1849. Overall, we recommend the Moving Average method, MA, when forecasting time series that follow lognormal distributions.

\section{DATA ANALYSIS}

In this study, we compare forecast precisions and accuracies from the forecasting methods Moving Average (MA) and Moving Median (MMd) on various skewed i.i.d. time series following lognormal distributions. Forecast precisions and accuracies are measured by absolute measures, $\bar{e}$ and $\mathrm{s}_{\mathrm{e}}$, and relative measures $\mathrm{L}_{\alpha}$ for $\alpha=0,0.2,0.4,0.6,0.8$, and, 1.0.

A lognormal probability distribution is a probability distribution for a continuous random variable $\mathrm{X}>0$, where $\ln (\mathrm{X})$ follows a normal probability distribution. In summary, when $\mathrm{X}$ follows a lognormal distribution and $\ln (\mathrm{X})$ follows a normal distribution with a mean of $\mu$ and a s.d. of $\sigma$, its probability density function for $\mathrm{X}$ is as follows

$$
\mathrm{f}(\mathrm{x})=\frac{1}{x \sigma \sqrt{2 \pi}} e^{-\frac{(\ln x-\mu)^{2}}{2 \sigma^{2}}}, \mathrm{x}>0
$$

with $\mathrm{E}(\mathrm{X})=e^{\left(\mu+\frac{\sigma^{2}}{2}\right)}, \operatorname{Median}(\mathrm{X})=e^{\mu}, \operatorname{Var}(\mathrm{X})=\left(e^{\sigma^{2}}-1\right) e^{\left(2 \mu+\sigma^{2}\right)}$, and the Skewness $=\left(e^{\sigma^{2}}+\right.$ 2) $\sqrt{e^{\sigma^{2}}-1}$

Examples of lognormal distributions can be found in the fields of finance, and economics (Johnson, Kotz, and Balakrishnan, 1994, PP. 210-211, and Antoniou, Ivanov, Ivanov, and Zrelov, 2004).

In this study, a simulation of 1,000 observations from a lognormal distribution are grouped into 50 groups each with 20 observations. For each group, the first 10 observations are treated as historical data, and the next 10 observations are treated as the realizations of actual observations. Moving averages (MA) and moving medians (MMd) with moving periods of 10 are applied as our forecasts for period 11 to 20 . Forecast errors are defined as the differences between the realizations of actual observations for period 11 to 20 and the forecasts generated from MA and MMd with moving periods of 10. The MA and MMd with moving periods of 10 are denoted as $\mathrm{MA}(10)$ and $\mathrm{MMd}(10)$, respectively.

Pairwise T-test (or the Student T-test) for testing $\mathrm{H}_{0}: \mu_{\mathrm{MA}} \geq \mu_{\mathrm{MMd}} \mathrm{vs}_{\mathrm{a}}: \mu_{\mathrm{MA}}<\mu_{\mathrm{MMd}}$ is applied to the

50 independent $\bar{e}, \mathrm{~s}_{\mathrm{e}}$, and $\mathrm{L}_{\omega}$ for $\alpha=0$ (for $\frac{s_{e}}{\bar{e}}$ ), 0.2, 0.4, 0.6, 0.8, and 1.0 (for $\frac{\bar{e}}{s_{e}}$ ) generated from MA(10) and $\mathrm{MMd}(10)$, respectively. The results for the T-test are listed on the following page.

\section{CONCLUSION}

In this study, we find that the Moving Median method, MMd, only outperforms the Moving Average method, MA, in terms of forecasting accuracy for time series follow a very skewed lognormal probability distribution with parameters $(\mu=0, \sigma=1)$, and skewness 6.1849. Overall, we recommend the Moving Average method, MA, when forecasting time series that follow lognormal distributions (e.g. stock prices). 


\section{TABLE 1}

TESTING $\mathrm{H}_{0}: \mu_{\mathrm{e}, \mathrm{MMd}} \geq \mu_{\mathrm{e}, \mathrm{MA}}$ vs $\mathrm{H}_{\mathrm{a}}: \mu_{\mathrm{e}, \mathrm{MMd}}<\mu_{\mathrm{e}, \mathrm{MA}}$ for LOGNORMAL with $\boldsymbol{\mu}=\mathbf{0}, \sigma=1$

\begin{tabular}{|c|c|c|c|c|c|c|c|c|}
\hline & $\overline{e_{1}}-\overline{e_{2}}$ & $s_{e_{1}}-s_{e_{2}}$ & $\begin{array}{c}\mathrm{L}_{\omega=0} \\
\frac{S_{e_{1}}}{\overline{e_{1}}}-\frac{S_{e_{2}}}{\overline{e_{2}}}\end{array}$ & $\mathrm{~L}_{\omega=0.2}$ & $\mathrm{~L}_{\omega=0.4}$ & $\mathrm{~L}_{\omega=0.6}$ & $\mathrm{~L}_{\omega=0.8}$ & $\begin{array}{c}\mathrm{L}_{\omega=1.0} \\
\frac{\overline{e_{1}}}{S_{e_{1}}}-\frac{\overline{e_{2}}}{S_{e_{2}}}\end{array}$ \\
\hline Mean & 0.4811 & -0.0693 & 2.5444 & 2.1086 & 1.6727 & 1.2369 & 0.8010 & 0.3652 \\
\hline s.d. & 0.3442 & 0.1182 & 34.0192 & 27.2158 & 20.4128 & 13.6106 & 6.8116 & 0.3643 \\
\hline T-ratio & 9.8833 & -4.1435 & 0.5289 & 0.5478 & 0.5794 & 0.6426 & 0.8315 & 7.0884 \\
\hline p-value & 1.0000 & $0.0001 *$ & 0.7004 & 0.7069 & 0.7175 & 0.7383 & 0.7951 & 1.0000 \\
\hline
\end{tabular}

* significant at $\alpha=0.01$, the skewness $=\left(e^{\sigma^{2}}+2\right) \sqrt{e^{\sigma^{2}-1}}=(\mathrm{e}+2) \sqrt{e-1} \approx 6.1849$

\section{TABLE 2}

TESTING $\mathrm{H}_{0}: \mu_{\mathrm{e}, \mathrm{MMd}} \geq \mu_{\mathrm{e}, \mathrm{MA}}$ vs $\mathrm{H}_{\mathrm{a}}: \mu_{\mathrm{e}, \mathrm{MMd}}<\mu_{\mathrm{e}, \mathrm{MA}}$ for LOGNORMAL with $\boldsymbol{\mu}=\mathbf{0}, \sigma=0.5$

\begin{tabular}{|c|c|c|c|c|c|c|c|c|}
\hline & $\overline{e_{1}}-\overline{e_{2}}$ & $s_{e_{1}}-s_{e_{2}}$ & $\begin{array}{c}\mathrm{L}_{\omega=0} \\
\frac{S_{e_{1}}}{\overline{e_{1}}}-\frac{S_{e_{2}}}{\overline{e_{2}}}\end{array}$ & $\mathrm{~L}_{\omega=0.2}$ & $\mathrm{~L}_{\omega=0.4}$ & $\mathrm{~L}_{\omega=0.6}$ & $\mathrm{~L}_{\omega=0.8}$ & $\frac{\mathrm{L}_{\omega}=1.0}{\overline{e_{1}}} \frac{\overline{e_{2}}}{S_{e_{1}}}-\frac{s_{e_{2}}}{S_{e^{2}}}$ \\
\hline Mean & 0.1130 & 0.0050 & 6.7375 & 5.4273 & 4.1171 & 2.8069 & 1.4967 & 0.1865 \\
\hline s.d. & 0.0953 & 0.0456 & 41.4876 & 33.1988 & 24.9101 & 16.6214 & 8.3332 & 0.1572 \\
\hline T-ratio & 8.3767 & 0.7757 & 1.1483 & 1.1560 & 1.1687 & 1.1941 & 1.2700 & 8.3866 \\
\hline p-value & 1.0000 & 0.7792 & 0.8718 & 0.8733 & 0.8759 & 0.8809 & 0.8950 & 1.0000 \\
\hline
\end{tabular}

* significant at $\alpha=0.01$, the skewness $\approx 1.9446$

TABLE 3

TESTING $\mathrm{H}_{\mathbf{0}}: \mu_{\mathrm{e}, \mathrm{MMd}} \geq \mu_{\mathrm{e}, \mathrm{MA}}$ vs $\mathrm{H}_{\mathrm{a}}: \mu_{\mathrm{e}, \mathrm{MMd}}<\mu_{\mathrm{e}, \mathrm{MA}}$ for LOGNORMAL with $\boldsymbol{\mu}=\mathbf{0}, \sigma=0.25$

\begin{tabular}{|c|c|c|c|c|c|c|c|c|}
\hline & $\overline{e_{1}}-\overline{e_{2}}$ & $s_{e_{1}}-s_{e_{2}}$ & $\begin{array}{c}\mathrm{L}_{\omega=0} \\
\frac{S_{e_{1}}}{\overline{e_{1}}}-\frac{S_{e_{2}}}{\overline{e_{2}}}\end{array}$ & $\mathrm{~L}_{\omega=0.2}$ & $\mathrm{~L}_{\omega=0.4}$ & $\mathrm{~L}_{\omega=0.6}$ & $\mathrm{~L}_{\omega=0.8}$ & 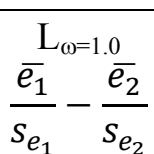 \\
\hline Mean & 0.0259 & -0.0010 & -0.7038 & -0.5451 & -0.3865 & -0.2278 & -0.0691 & 0.0896 \\
\hline s.d. & 0.0384 & 0.0167 & 23.3133 & 18.6559 & 13.9986 & 9.3414 & 4.6849 & 0.1397 \\
\hline T-ratio & 4.7810 & -0.4333 & -0.2135 & -0.2066 & -0.1952 & -0.1724 & -0.1043 & 4.5343 \\
\hline p-value & 1.0000 & 0.3333 & 0.4159 & 0.4186 & 0.4230 & 0.4319 & 0.4587 & 1.0000 \\
\hline
\end{tabular}

* significant at $\alpha=0.01$, the skewness $\approx 0.8340$

TABLE 4

TESTING $\mathrm{H}_{0}: \mu_{\mathrm{e}, \mathrm{MMd}} \geq \mu_{\mathrm{e}, \mathrm{MA}}$ vs $\mathrm{H}_{\mathrm{a}}: \mu_{\mathrm{e}, \mathrm{MMd}}<\mu_{\mathrm{e}, \mathrm{MA}}$ for Lognormal with $\mu=0, \sigma=0.1$

\begin{tabular}{|l|c|c|c|c|c|c|c|c|}
\hline & $\overline{e_{1}}-\overline{e_{2}}$ & $s_{e_{1}}-s_{e_{2}}$ & $\begin{array}{c}\mathrm{L}_{\omega=0} \\
\frac{s_{e_{1}}}{S_{e_{2}}}\end{array}$ & $\mathrm{~L}_{\omega=0.2}$ & $\mathrm{~L}_{\omega=0.4}$ & $\mathrm{~L}_{\omega=0.6}$ & $\mathrm{~L}_{\omega=0.8}$ & $\frac{\mathrm{L}_{\omega=1.0}}{\overline{e_{2}}}$ \\
& & & & & & & $\frac{\overline{e_{1}}}{s_{e_{1}}}$ \\
\hline Mean & 0.0048 & 0.0002 & -59.8470 & -47.866 & -35.886 & -23.906 & -11.926 & 0.0543 \\
\hline s.d. & 0.0152 & 0.0049 & 316.020 & 252.817 & 189.613 & 126.410 & 63.2067 & 0.1723 \\
\hline T-ratio & 2.2445 & 0.2284 & -1.3391 & -1.3388 & -1.3383 & -1.3373 & -1.3342 & 2.2282 \\
\hline p-value & 0.9853 & 0.5899 & 0.0934 & 0.0934 & 0.0935 & 0.0937 & 0.0942 & 0.9848 \\
\hline
\end{tabular}

* significant at $\alpha=0.01$, the skewness $\approx 0.3113$ 


\section{REFERENCES}

Anderson, D., Sweeney, D., Williams, T., Camm, J., \& Cochran, J. (2016). Modern Business Statistics with Microsoft Office Excel. $6^{\text {th }}$ ed., Boston, MA: Cengage Learning.

Antoniou, I., Ivanov, Vi., Ivanov Va., \& Zrelov, P. (2004). On the log-normal distribution of stock market data. Physica A, 331, 617-638.

Armstrong, J., \& Collopy, F. (1992). Error measure for generalizing about forecasting methods: empirical comparisons. International Journal of Forecasting, 8, 69-80.

Baldwin, M. E., \& Kain, J. S. (2006). Sensitivity of several performance measures to displacement error, bias, and event frequency. Weather \& Forecasting, 21(4), 636-648.

Chatfield, C. (1998). Apples, oranges, and mean squared error. International Journal of Forecasting, 4, 515-518.

Chen, C., Twycross, J., \& Garibaldi, J. M. (2017). A new accuracy measure based on bounded relative error for time series forecasting. Plos ONE, 12(3), 1-23.

Flores, B. E., \& Wichern, D. W. (2005). Evaluating forecasts: a look at aggregate bias and accuracy measures. Journal of Forecasting, 24(6), 433-451.

Hoover, J. (2006), Measuring forecast accuracy: omissions in today's forecasting engines and demandplanning software. Foresight, 4, 32-35.

Johnson, N., Kotz, S., \& Balakrishnan, N. (1997). Continuous Univariate Distributions, Ch. 14(1), $2^{\text {nd }}$ ed., New York, New York: John Wiley \& Sons.

Limpert, E., Stahel, W, \& Abbt M. (2001). Lognormal distributions across the sciences: keys and clues. BioScience, 51(5), 341-352.

Makridakis S. (1993). Accuracy measures: theoretic and practical concerns. International Journal of Forecasting, 9, 527-529.

Mazais, J., Miķelsons, J., \& Salenieks, N. (2007). Process parameter and measurement accuracy and precision. Transport \& Engineering, 2311-15.

Morlidge, S. (2016). Using error analysis to improve forecast performance. Foresight: International Journal of Applied Forecasting, 41, 37-44.

Shang, H. L. (2015). Statistically tested comparisons of the accuracy of forecasting methods for agespecific and sex-specific mortality and life expectancy. Population Studies, 69(3), 317-335. 